98 Heintze: Ein Beitrag zur Geschichte des Ultramarins.

$$
\mathrm{C}_{10} \mathrm{H}_{7} \mathrm{OH}+\left(\mathrm{CH}_{9}\right)_{2} \mathrm{CHCOOH}=\mathrm{C}_{10} \mathrm{H}_{8}(\mathrm{OH}) \mathrm{CO} \cdot \mathrm{CH}\left(\mathrm{CH}_{3}\right)_{2}+\mathrm{H}_{2} \mathrm{O}
$$

gebildet hat und das mit Aether und Alkohol ausgezogen und gereinigt, blassgelbliche, aromatisch riechende, luftbeständige Krystalle darstellt, deren Schmelzpunkt bei $79^{\circ}$ liegt.

$0,233 \mathrm{Grm}$. dieses Ketons gaben 0,6668 Grm. $\mathrm{CO}_{2}$ und 0,143 Grm. $\mathrm{H}_{2} \mathrm{O}=78,06 \% \mathrm{C}$ und $6,82 \%$ H. Die obige Formel verlangt $78,5 \% \mathrm{C}$ und $6,54 \% \mathrm{H}$.

\title{
Ein Beitrag zur Geschichte des Ultramarins;
}

von

\section{J. Heintze.}

Die Geschichte der Erfindung des künslichen Ultramarins ist, wenn auch schon weit zurückliegend, nicht ohne ein gewisses dramatisches Interesse. Dieses Problem beschäftigte die forschenden Chemiker jener Zeit in hohem Grade, und es erregte allgemeines und berechtigtes Aufsehen, als GayLussac in der Sitzung des französischen Institutes am 4. Februar 1828 mittheilte, dass es Guimet gelungen wäre, nach den Analysen von Désormes und Clement, künstliches Ultramarin von verschiedenen Sorten herzustellen. Infolge dieser Mittheilung veröffentlichte Gmelin in der Spener'schen Zeitung vom 10. April 1828, dass er schon seit längerer Zeit mit der Untersuchung des Ultramarins beschäftigt sei, es auch gegen Gay-Lussac im Frühjahr 1827 persönlich ausgesprochen, dass er sich mit diesen Untersuchungen beschäftige und dadurch vielleicht indirect die Schuld trage, dass $\mathrm{ihm}$ ein Anderer (Guimet) mit dieser Entdeckung zuvorgekommen sei. Guimet hat seine Methode geheim gehalten, erhielt aber im Jahre 1828 von der Société d'encouragement den von dieser Gesellschaft gestifteten Preis für die gelungene Darstellung des Ultramarins. Gmelin veröffentlichte bald sein Verfahren in eingehender Beschreibung. Während so beide Erfinder mit den Resultaten ihrer Forschungen vor die Oeffentlichkeit traten, hatte sich ein dritter Chemiker, unabhängig von jenen, gleich- 
falls erfolgreich mit der Lösung dieser Aufgabe beschäftigt, und da er es unterliess, seine Erfolge seiner Zeit zu publieiren, hat diese Thatsache überall in der chemischen Literatur eine nur ungenaue Darstellung gefunden. - Nahezu gleichzeitig mit Guimet und Gmelin war es auch Friedrich August Köttig, Vorstand des Laboratoriums der Königl. Porzellan-Manufactur in Meissen, gelungen, das Ultramarin nach einer für den Fabrikbetrieb geeigneten Methode herzustellen. Wenn dann auch an ungeeigneten Orten ${ }^{1}$ ) versucht worden ist, Köttig's Antheil an dieser Erfindung in Anspruch zu nehmen, so sind diese Anzeigen doch nicht in die wissenschaftliche Literatur gelangt und daher nicht beachtet worden, So sagt z. B. Otto in seinem Lehrbuch der angewandten Chemie, dass zwar Guimet den ausgesetzten Preis erhalten habe, es aber Gmelin schon früher gelungen sei, Ultramarin künstlich zu bereiten. Otto fährt dann ferner fort: „Engelhard, Professor der Chemie in Nürnberg, der Uebersetzer von Dumas' Werken, veranlasst durch einen Hinweis von $\mathrm{Du}$ mas, stellte Versuche an, das künstliche Ultramarin ebenfalls herzustejlen. Er starb, ehe er sein Ziel erreichte, aber sein Assistent und Nachfolger Leykauf setzte die Versuche fort, und ihm zuerst gelang die massenhafte Fabrikation der Farbe. Einige Zeit darauf fabricirte auch die Porzellan-Manufactur in Meissen vortreffliches Ultramarin." Otto ist hier nicht genau unterrichtet, denn Köttig fabricirte in Meissen bereits seit Frühjahr 1829 seine vortrefflichen Ultramarinfarben, während Leykauf erst im Jahre 1836 auftrat. Gmelin veröffentlichte in den Naturwissenschaftlichen Abhandlungen einer Gesellschaft in Württemberg, Jahrgang 1828, Bd. 2, das Verfahren, durch welches ihm die Darstellung des Ultramarins immer gelingt. Fr führt nämlich an, dass er diese Verbindung durch Auflösung von Kieselsäure in kaustischem Natron, unter berechnetem Zusatz von Thonerde und Abdampfen, anderseits durch Schmelzen von kohlensaurem Natron und Schwefel, durch Eintrag der ersteren Masse in kleinen Portionen in die Letztere, schliessliches, starkes Glühen bei Rothgluth und durch endliches Abschlämmen der erhaltenen Fritte erhalten habe.

1) Illustrirte Zeitung, Jahrgang 1851, Nr. 437. 
100 Heintze: Ein Beitrag zur Geschichte des Ultramarins.

Köttig hatte sich im Frühjahr 1828 mit der Herstellung einer flüssigen, bleifreien Glasur beschäftigt, und als er zu diesem Zwecke ein Natronsilicat gebildet hatte, zu dem er einen reinen Thon zusetzte, fand er nach dem Glühen eine blaue, erdige Substanz in der Fritte eingesprengt. Nach Wiederholung dieses Versuchs und nach dem Studium der gr genseitigen Einwirkung der angewendeten Stoffe, nämlich schwefelsauren Natrons, Quarzpulver, Holzkohle und Thon, erhielt er nach dem Glühen stets einen blauen Farbkörper. Da man aus den erwähnten Analysen wusste, dass das Ultramarin aus Thonerde, Kieselsäure, Natron und Schwefel bestand, in der neuen Farbe aber dieselben Verbindungen enthalten waren, so war es Köttig sofort klar, in der blauen Farbe künstliches Ultramarin gefunden zu haben. Er ging nun weiter und begann eine grössere Menge der neuen Farbe $z u$ fabriciren. Da erschien im Jahrbuch für Chemie und Physik von Schweiger-Seidel, Jahrgang 1828, Bd. 1, Heft II, die Abhandlung von Gmelin, in welcher derselbe die Resultate seiner schon erwähnten Arbeit über Ultramarin in eingehender Weise entwickelte. Köttig fand hier seine Erfahrungen voll bestätigt, jedoch kann man wohl sagen, dass das Verfahren von Köttig insofern das sicherere war, weil er seinem Gemisch Holzkohlenpulver zusetzte, denn erst durch die reducirenden Wirkungen der Kohle wurde das Verfahren sicher und konnte $\nabla \cap n$ einer zuverlässigen Production die Rede sein.

Im October 1828 hatte Köttig seine Versuche beendet, und nach den nöthigen Vorverhandlungen begann bereits in den ersten Monaten des Jahres 1829 die Lasursteinblaufarbenfabrik der Königl. Porzellan-Manufactur zu Meissen die neuen Farben in den Handel zu bringen. Nahezu ein halbes Jahrhundert ist die Fabrikation schwunghaft betrieben worden, bis die Fabrik aus äusseren Gründen Ende der siebziger Jahre geschlossen und der Betrieb eingestellt wurde.

Bei dieser Gelegenheit stellte sich erneut heraus, dass sich diese Farben bei einer grossen Zahl von Abnehmern einer bedeutenden Schätzung erfreuten und sie vielfach den Produkten anderer Fabriken vorgezogen wurden. Es ist daher vielleicht noch heute ron Interesse, das Verfahren kennen zu lernen, nach welchem die Herstellung der Meissner Ultra- 
marinfarben im Grossen erfolgt ist. Es hat heute kein Interesse mehr, die Stadien der Entwickelung zu beschreiben; die Fabrikation geschah anfangs nur in relativ kleinen Verhältnissen, bis es später gelang, in grossem Maassstabe zu arbeiten. - Die Anwendung von schwefelsaurem Natron stellte sich bald als unzweckmässig heraus, und man bediente sich des chemisch reinen krystallisirten kohlensauren Natrons, da sich zeigte, dass schon geringe Verunreinigungen der Soda von schwefelsauren und schwefligsauren Salzen die Lebhaftigkeit der Farbe beeinträchtigten. Auch fand man, dass nicht jeder reine Thon oder Kaolin geeignet war, weil das procentale Verhältniss von Thonerde zur Kieselsäure von Bedeutung war, z. B. hatte die Porzellanerde von Morl bei Halle einen zu hohen Kieselsäuregehalt und man erzielte mit dem rein geschlämmten Kaolin von Seilitz, der englischen Porzellanerde, sogenanntem China-Clay und dem Kaolin von Zettlitz bei Karlsbad in Böhmen die besten Resultate. - Man verwendete ferner sicilianischen Stangenschwefel, der auf Kugelmühlen mittelst eiserner Kugeln zu einem feinen Pulver gemahlen wurde; ebenso wurde kieferne und fichtene Holzkohle angewendet, die auf Kugelmühlen oder Kollergängen fein pulverisirt wurde.

Der eigentliche Gang der Fabrikation begann mit der Bildung einer Grundmasse, indem 50 Theile krystallisirtes, chemisch reines kohlensaures Natron und 20 Theile geschlämmtes Kaolinpulver abgewogen wurden. Die Soda gelangte in entsprechend grosse, gusseiserne Schalen von der Form der Abdampfschalen von etwa 1 Meter Durchmesser und 0,3 Meter Tiefe, unter welcher sich eine directe Feuerung befand. Die Soda beginnt bald in ihrem Krystallwasser zu schmelzen, und in die kochende Schmelze wird die genannte Menge Kaolin, zwar in kleinen Portionen, aber doch schnell eingetragen und bei schwachem Feuer unter stetigem Rühren nit einem eisernen Rührscheit bis zur Trockne rerdampft. Durch diese Operation erhält man die sogenannte Farbebasis, etwa 32 Theile der angewandten Mengen. Auf 19 Theile dieser Schmelze setzt man 6 Theile Schwefelpulver und 1 Theil Holzkohlenpulver. Es giebt dieses Verhältniss nach dem Brande ein schönes Blau, Beschickungen von vermindertem 
Schwefelzusatz geben ein helleres Blau. Erniedrigt man z. B. den Schwefelzusatz von 6 auf 4 Theile, so erhält man nicht den röthlichen, sondern einen grünen Ton. Einen ähnlichen Erfolg erzielt man auch durch Vermehrung des Natronzusatzes, wenn man z. B. 40 Theile Kaolin, 132 Theile Soda wählt, wozu bei 85 Theilen Basis 24 Theile Schwefel und 5 Theile Kohle gemengt werden. Es lassen sich durch wechselnde procentale Verhältnisse verschiedene Nüancen der Ultramarine erzielen. Die vorstehenden Farbenmischungen werden nun in Kugelmühlen von entsprechender Grösse mittelst Porzellankugeln bei einer mässigen Umdrehungsgeschwindigkeit ca. 10 Stunden gemischt. Die innigste Mischung ist eine wesentliche Vorbedingung des Gelingens der folgenden Processe; man kann sich von der richtigen Ausführung leicht überzeugen, wenn das erhaltene Pulver, aufgestrichen, keine Einzelbestandtheile inehr erkennen lässt. Das gemischte Produkt gelangt nun in offene, flache, runde Kapseln aus Chamotte, und wird jede Kapsel mit 250 Grm. Masse beschickt, so dass sie $\mathrm{zu}^{3} / 4$ gefüllt, und nachdem man das Pulver etwas eingeklopft hat, eine 3-4 Cm. hohe Schicht entstanden ist. Man pflegt etwa 50 Kilogrm. Masse in 200 bis 202 Kapseln zu vertheilen. Die Kapseln wurden nun zum Rohbrand in Muffelöfen eingesetzt; man bediente sich hierzu Muffelöfen, welche paarweise übereinander gelegt waren. Die Muffeln fassten etwa $0,4 \mathrm{Cbm}$. und wurden meistens $216 \mathrm{Kapseln}$ in 24 gleichen Stössen à 9 Kapseln Höhe und in 4 Reihen a 6 Stössen neben einander eingesetzt, wodurch die Muffel vollständig gefüllt wurde. In der späteren Fabrikation warf man für diesen Brand allerdings die Kapselfüllung ab und beschickte den Ofen mit 90 Chamottetiegeln von je 1,8 Lit. Capacität, die ebenfalls mit Deckeln abgedeckt wurden, um in dreifacher Höhe aufgebaut $\mathrm{za}$ werden. Die übrigen Muffelöfen wurden analog besetzt. Man benutzte auch die Muffelöfen ohne besonders eingezogene Muffel, deckte dann auf die durch einen passenden Deckel verschlossenen oberen Kapseln oder Tiegel grosse Chamotteplatten, um die Flamme zu zwingen, die Farbestösse von allen Seiten zu umspülen, wobei man zwischen den einzelnen Stössen einige Centimeter Feuergasse bildete. Um ein gutes Fabrikat zu erhalten, ist es von Wichtigkeit, weder eine zu starke noch zu schwache Glühung der 
Farben vorzunehmen. Der mit einer Mauer zugesetzte Ofen, welcher durch eingesetzte Rohre mit Blechschiebern beobachtet werden kann, wird mit Kohlen gefeuert, und ist es zweckmässig, auf einen vollen, reducirenden, sauerstofffreien Flammengang zu halten. Aus den geschlossenen Gefässen entweichen während des Brandes Kohlenoxyd und schweflige Säure. Der Rohbrand wird beendigt, sobald der Inhalt des Ofens bis zur Temperatur der Gelbglühhitze gleichmässig erhitzt worden ist, bei normalen Gang vach $2^{1} / 4$ bis $2^{1 / 2}$ Stunden. Zur Erzielung einer längeren Nachgluth ist es von Vortheil, nach Beendigung des Brandes mittelst eines zugeschobenen Thonschiebers den Essenzug abzusperren. Nach dem Abkühlen des Ofens und Entleeren der Kapseln erhält man die wenig geschwundene, blau oder bläulichgrau gefärbte Rohfarbe als leicht zerreibliche Masse.

Der Brand ist gelungen, wenn die Farbemasse innerlich durch und durch gleichmässig gefärbt und dabei so locker und weich ist, dass man sie zwischen den Fingern leicht zerreiben kann. Hat sie zuwenig Feuer erhalten, so erscheint sie entweder theilweise oder noch durchgängig grau. Sie ist dann zur weiteren Bearbeitung unbrauchbar. War dagegen der Brand zu scharf, so ist die Farbemasse mehr geschwunden, die entstandenen Rohfarbescheiben sind gerissen, und obgleich die Masse normal gefärbt erscheint, ist sie wegen der eingetretenen Sinterung zu hart und liefert keine schöne blane Farbe. Aus der erhaltenen Rohfarbe sind nun die überschüssigen, löslichen Salze verschiedener Schwefelungsstufen durch Auswaschen zu entfernen. In entsprechenden Auslaugbütten wird sie daher zunächst mit heissem, später mit kaltem Wasser so lange ausgewaschen, bis das $W$ aschwasser keinen merklichen Rückstand hinterlässt. Der Farbeschlamm wird dann abgepresst, in Kapseln auf Trockenöfen getrocknet und durch ein mittelfeines Messingdrahtsieb gesiebt. War die Farbe richtig und nicht zu hart gebrannt worden, ist sie so beschaffen, dass sie leicht mittelst Hand durch das Sieb getrieben werden kann. Die Farbe bedarf nun noch eines zweiten Brandes, des Ausglühbrandes. Derselbe hat den $Z_{w e c k}$, gewisse grünliche Schwefelverbindungen, welche noch in der Farbe enthalten sind, durch eine zweite Glühung bei Gegenwart von Sauerstoff zu zersetzen, 
wodurch eine rein blaue Farbe erzielt wird. Die Farbe wird, in ähnlicher Weise wie bei dem ersten Brand besprochen, in Kapseln gefült und eingesetzt, nur sind an den Kapseln drei Ausschnitte angebracht, um der Luft freien Zutritt zu gestatten. Auch müssen nunmehr die Farben vor der Verunreinigung durch Flugasche geschützt werden, und man nimmt daher die Glühung in geschlossenen Muffeln vor, welche etwa je 0,35 Cbm. Fassungsraum besitzen. Die Muffel wird sonst in gleicher Weise geschlossen, mit zwei Schaurohren versehen und mit Kohlen gefeuert, wie bei dem Rohbrand ausgeführt. Da die Kapseln vor der directen Einwirkung der Flammen geschützt sind, ist in der Muffel eine stark oxydirende Atmosphäre. Die Temperatur des Brandes wird bis zur mässigen Rothgluth gesteigert. Es erfordert dieser Brand ziemliche Aufmerksamkeit, weil der Feuersgrad sich nach dem vorhergegangenen Rohbrand zu richten hat und daher relativ schwankend ist; denn war die Farbe im Rohbrand härter gebrannt, muss auch die Temperatur des Ausglühbrandes etwas gesteigert werden. Zu weiche Rohbrände besitzen an sich schon eine lichte, grün graublaue Farbe und dürfen daher nur schwach, kaum dunkelroth geglüht werden, um noch ein brauchbares Fabrikat zu liefern. Bei Beginn des Glühens nimmt die Rohfarbe bald eine dunkle, fast schwarze Farbe an, die sich nach und nach in Blau verwandelt. In dieser Uebergangsperiode, noch bevor das reine Blau völlig eingetreten ist, muss die Glühung beendet werden. Die Nachgluth, welche, wie schon früher besprochen, durch Schluss des Essenschiebers verlängert wird, wirkt noch besonders günstig auf die Bildung des röthlichen oder violetten Farbetones ein. Der Ausglühbrand dauert etwa $2 \frac{1}{2}$ Stunde.

Mit dem Ausglühen des Ultramarins sind die eigentlich chemisch-technischen Arbeiten beendigt, und es bedarf nur noch einiger mechanischen Arbeiten und zwar des Feinmahlens und Schlämmens der fertigen Farbe. Es sollen daher die weiteren Manipulationen nur noch kurz besprochen werden. Mengen von 25 Kilogrm. gebrannter Farbe werden mit Wasser angerührt, und pflegte man bei den feineren Qualitäten einen Zusatz von 100 Grm. kohlensaurer Magnesia, oder bai geringeren von $2 \breve{0} 0 \mathrm{Grm}$. gemahlener Kreide zuzumischen, wodurch während des Auswaschens eine schnellere Klärung erzielt wurde. Vor dem 
Auswaschen wurde aber die Farbe in Kübelmühlen noch 24 Stunden mit dem nothwendigen Wasser sehr fein gemahlen. Das Mahlgut wird endlich durch feine Haarsiebe gegossen und durch wiederholtes Auswaschen von noch löslichen Salzen und wiederholtes Abschlämmen von noch vorhandenen gröberen Theilen getrenut. In Folge der sehr feinen Mahlung der Ultramarinfarben verliert nun allerdings die ausgeglühte, schöne dunkelfeurige Farbe viel von ihrem Ansehen, und aus diesem Grunde kommen noch heute im Handel nicht sehr fein gemahlene Farben vor; berücksichtigt man aber, dass die Ultramarine nicht nur als Malfarbe, sondern hauptsächlich als Bläuungsmittel, als Druckfarbe bei Geweben etc. Verwendung finden, wo auf eine ausserordentlich feine Vertheilung besonderer Werth zu legen ist, so ist die feine Mahlung ebenso wichtig als ein dunkler Ton und es mag an diesen Eigenschaften gelegen haben, dass die Köttig'schen Ultramarine lange Zeit eine grosse Zahl stabiler Abnehmer aus der Zahl der Grossindustrie hatten. Um beide Bedingungen, Feinheit und Intensität, zu vereinigen, ist es daher nothwendig, bereits im Rohbrand ein Präparat zu erzielen, welches bei gleichförmig dunkler und kräftiger Färbung in einem weichen Aggregatzustande sich befindet. In dieser Beziehung hat auch das Meissner Ultramarin während seiner Fabrikation vielfach den Vorzug vor den Farben anderer Fabriken behauptet, deren dunkelste und schönste Nüancen den Köttig'schen an Feinheit und Ergiebigkeit nachgestanden haben. Die besseren Marken sind die reinen Farbekörper, während die billigeren Sorten noch im nassen Zustande mit Kaolinschlamm verdünnt wurden. Die fertigen Farben werden schliesslich getrocknet und durch feine Siebe cylindert. Es wurden schliesslich blaue und grüne Ultramarinfarben in den Handel gebracht, welch' letztere, in die Tiegel fest eingestampft, durch die Glühung im Rohbrand bei möglichstem Luftabschluss sich leicht bilden.

Bieten vorstehende Mittheilungen auch nur einiges Neue, so dürften sie doch immerhin Interesse verdienen, da sie auf den Erfahrungen eines langjährigen erfolgreichen Grossbetriebes beruhen.

Meissen, am 14. November 1890. 